\title{
KOSTENRECHNUNG UND NEOINSTITUTIONELLE BETRACHTUNGSWEISE: ÖKONOMISCHE BEGRÜNDUNGSANSÄTZE VON FIX- UND GEMEINKOSTENALLOKATIONEN
}

\section{Dieter Pfaff, Johann Wolfgang Goethe-Universität Frankfurt a.M.}

Gegenstand des Übersichtsvortrags ist die ökonomische Begründung von Verfahren der Fix- und Gemeinkostenallokation. Einerseits werden in der "traditionellen" Theorie häufig Verfahren der Vollkostenrechnung abgelehnt. Andererseits konnten sich Teilkostensysteme in der Praxis nicht hinreichend durchsetzen. Diese Diskrepanz läßt sich insbesondere damit begründen, daß die in der Theorie gewonnenen Erkenntnisse auf der Annahme eines einzigen (in der Regel risikoneutralen) Entscheidungsträgers beruhen, der über alle entscheidungsrelevanten Informationen verfügt. In realen Organisationen dagegen herrschen dezentrale Entscheidungssysteme vor, in denen Informationen asymmetrisch verteilt sind und die einzelnen Entscheidungsträger ihren Informationsvorsprung im eigenen Interesse und nicht im Sinne der vorgesetzten Instanzen nutzen. Sie geben u.a. auch Fehlinformationen, um die Entscheidungen dieser Instanzen im eigenen Interesse zu beeinflussen.

Erkenntnisse, die in einer "idealen" Welt eines einzelnen wohlinformierten Entscheidungsträgers gewonnen werden, sind damit nicht ohne weiteres auf Organisationen übertragbar. Die Mehrzahl der in der Fachliteratur diskutierten ökonomischen Begründungsansätze von Fix- und Gemeinkostenallokationen läßt sich unter vier Forschungsschwerpunkten zusammenfassen: Präferenz, Dekomposition, Anreiz und Koordination. Im ersten Fall hängt die Entscheidungsrelevanz von Fixkosten nur von der Gestalt der subjektiven Präferenzen des Entscheidungsträgers - üblicherweise seiner Risikoeinstellung - ab. Alle Präferenzmodelle betrachten einen einzigen Entscheider mit lediglich einem Entscheidungsproblem in einem Unternehmen. Demgegenüber gibt es bei den Dekompositionsansätzen zum Teil mehrere Entscheidungsträger, zumindest aber mehr als ein Entscheidungsproblem. Dabei wird die Fähigkeit von Gemeinkostenallokationen analysiert, die regelmäßig auftretenden Interdependenzen zwischen den einzelnen Entscheidungsproblemen zu approximieren. Unterstellt man zusätzlich zu den Annahmen der Dekompositionsmodelle asymmetrische Informationsverteilung und opportunistisches Verhalten, kommt man zu Anreiz- und Koordinationsansätzen. Erstere untersuchen, ob die Zurechnnung von Gemein- oder Fixkosten das Verhalten eines Agents so beeinflussen kann, daß sich der Nutzen des Principals erhöht. Letztere behandeln die Allokationswirkungen auf mindestens zwei Agents, wodurch im Unterschied zum einfachen Anreizmodell zusätzlich Abstimmungs- und Risikoverteilungsprobleme zwischen den Agents relevant werden können. 